\title{
Improving Communication in Risk Management of Health Information Technology Systems by means of Medical Text Simplification
}

\author{
Silvana Togneri MacMahon \\ Department of Visual and Human \\ Centred Computing \\ Dundalk Institute of Technology \\ Dublin Road, Dundalk, Ireland \\ silvana.macmahon@dkit.ie \\ Giosuè Lo Bosco \\ Dipartimento di Matematica e \\ Informatica \\ Università di Palermo, Italy \\ giosue.lobosco@unipa.it
}

\author{
Marco Alfano \\ School of Computing \\ Dublin City University \\ Glasnevin, Dublin 9, Ireland \\ marco.alfano@dcu.i \\ Fergal McCaffery \\ Department of Visual and Human \\ Centred Computing \\ Dundalk Institute of Technology \\ Dublin Road, Dundalk, Ireland \\ fergal.mccaffery@dkit.ie \\ Markus Helfert \\ School of Computing \\ Dublin City University \\ Glasnevin, Dublin 9, Ireland \\ markus.helfert@dcu.ie
}

\author{
Biagio Lenzitti \\ Dipartimento di Matematica e \\ Informatica \\ Università di Palermo, Italy \\ biagio.lenzitti@unipa.it \\ Davide Taibi \\ Istituto per le Tecnologie Didattiche \\ Consiglio Nazionale delle Ricerche, \\ Palermo, Italy \\ davide.taibi@itd.cnr.it
}

\begin{abstract}
Health Information Technology Systems (HITS) are increasingly used to improve the quality of patient care while reducing costs. These systems have been developed in response to the changing models of care to an ongoing relationship between patient and care team, supported by the use of technology due to the increased instance of chronic disease. However, the use of HITS may increase the risk to patient safety and security. While standards can be used to address and manage these risks, significant communication problems exist between experts working in different departments. These departments operate in silos often leading to communication breakdowns. For example, risk management stakeholders who are not clinicians may struggle to understand, define and manage risks associated with these systems when talking to medical professionals as they do not understand medical terminology or the associated care processes. In order to overcome this communication problem, we propose the use of the "Three Amigos" approach together with the use of the SIMPLE tool that has been developed to assist patients in understanding medical terms. This paper examines how the "Three Amigos" approach and the SIMPLE tool can be used to improve estimation of severity of risk by non-clinical risk management stakeholders and provides a practical example of their use in a ten step risk management process
\end{abstract}

Keywords-Risk Management, IEC 80001-1, Medical Terminology Simplification

\section{INTRODUCTION (HEADING 1)}

HITSs have been recognised for their potential to improve patient care including reducing the instances of adverse events, improving patient safety, reducing the time spent by clinicians manually entering information, reducing redundant testing due to inaccessible information, improving patient care, reducing healthcare costs and ensuring comprehensive and secure management of health information [1]-[3]. The number of networked medical devices in use continues to increase as a result of these changes in models of care [4]-[6]. The use of proprietary networks is being phased out, as their use may limit the communication of the devices and therefore the potential benefits of connecting devices. This means that medical device manufacturers no longer exercise full control over the configuration of the network [7]. Hospitals regularly source network components and devices from different manufacturers creating a new system in which the device has not been validated [8], [9]. This can lead to risks which result in unintended consequences outside the control of the medical device manufacturer.

These risks can result in the incorrect and degraded performance of the medical devices compromising patient safety, effectiveness of the medical device and the security of the IT network. Standards have been developed and continue to be developed to ensure that the risks associated with these systems are managed. However, their adoption and usage is hampered by challenges in communication between different stakeholders along the standardisation process

The aim of this paper is to describe the communication process within the IEC 80001-1 standard [10]. The standard outlines the roles, responsibilities and activities related to the risk management of medical IT networks. We focus on examining limitations related to the communication among the different risk management stakeholders due to the difficulty of non-clinicians in understanding medical terminology. We also examine how communication in risk management can be improved by using a proven communication approach - the "Three Amigos" approach. By introducing SIMPLE, - a medical text simplification system, we aim to facilitate a better understanding of risk among non-clinical risk management stakeholders 
The remainder of the paper is structured as following. Section 2 presents the background and how standards are used to perform risk management of these systems. Subsequently, Section 3 introduces the "Three Amigos" approach together with the SIMPLE system and an example of their use in a riskmanagement scenario. Finally, as this research is at an early stage, Section 4 presents future work in this area and explains how this research leverages prior research conducted in this area by the authors.

\section{BACKGROUND AND RISK MANAGEMENT OF HITS}

\section{A. Risk Management Standard of HITS}

In order to address the risks of HITS, IEC 80001-1 was published in 2010 [10]. As part of the risk management activities, the standard defines a risk management process that should be followed by Healthcare Delivery Organizations (HDOs) throughout the lifecycle of the medical IT network. This process is carried out within the overall risk management of the HDO which will look at the wider risk context of the $\mathrm{HDO}$, such as the legal and reputational implications for the HDO in the event of a malpractice lawsuit.

During risk analysis, hazards are identified and the risks associated with these hazards are evaluated. Risk evaluation focuses on determining whether the identified risk is so low as to not require the use of risk control measure or whether risk control measures are required to ensure that the risk falls within risk acceptability criteria as defined in the risk management plan. During risk control, proposed risk control options are identified and documented for each unacceptable risk.

Where risk control measures are not available, a risk benefit analysis is to be conducted to assess the risk against the benefit of the use of the device to the patient. Risk control measures are implemented under the change-release management process defined in the standard and documented. Once implemented the effectiveness of the risk control measures are verified and analysis is conducted to ensure that no new risks have been introduced as a result of the introduction of the risk control measures. Residual risk analysis and reporting focuses on ensuring that individual residual risk and overall residual risk fall within the risk acceptability criteria as defined in the risk management plan.

The risk management process is focused on ensuring that the key properties of the network i.e. safety, effectiveness and security are maintained in order to ensure a high standard of care is provided to the patient. As the discussion of risk involves multidisciplinary stakeholders, arriving at a common understanding of risk and impact can be challenging. This is discussed further in subsection $\mathrm{C}$ of this section.

\section{B. Implementation and Adoption Challenges}

IEC 80001-1 is currently being revised and prior to the commencement of the revision work, a survey was conducted by the developers of the standard, among HDOs to assess their experience of implementing the requirements of IEC 80001-1.
The survey outlined a number of barriers to the adoption of the standards as follows:

1. Lack of drivers to motivate Top Management to implement the IEC 80001-1 standard;

2. HDO Organizational challenges: Information Technology (IT) and Biomedical Engineering (BME) departments are not aligned;

3. The IEC 80001-1 standard is too complicated and complex to implement.

ISO 31000 is not itself a management system standard but follows the format set out in Annex SL of the ISO Directives [11]. Adopting a similar approach allows the requirements of IEC 80001-1 to be integrated with those existing management system standards. This increases the drivers for management to adopt the standard as it allows for easier adoption of IEC 80001-1, leverages existing processes with the HDO and allows IEC 80001-1 to be revised in a form which is less complex and complicated. While this approach may address barriers 1 and 3 identified above, it does not directly address barrier 2 - the organizational challenges which are presented by a lack of alignment between IT and biomedical engineering (BME) departments within HDOs. It should be noted that in this paper clinical engineering and BME are used interchangeably.

The survey revealed that while there has been a move within hospitals to promote greater levels of communication between the clinical departments, and the IT departments, these departments still tend to operate in silos often leading to communication breakdowns. The feedback indicated that, in general, IT do not understand clinical workflows or that network connectivity has become a crucial element of patient care. It was also reported that IT management lack knowledge of basic risk management concepts such as safety and reliability engineering and Failure Mode Cause and Effect Analysis (FMCEA). While taxonomies of medical devices exist, for risk management purposes they do not provide context in terms of the clinical workflows in which these devices are used or the acuity of patients being treated. It was stated that "this resonates as the single largest impediment to 80001 adoption and needs clear and concise focus in the revision". It is also reported that BMEs do not understand complex networking concepts. They "do not speak the same language" [12].[13]. For example, when discussing the impact of a network outage, the IT department considered the impact from the perspective of downtime of the network while BME stakeholders considered the impacts that the outage may have while focusing primarily on patient safety but also on the operational impact that the downtime may have. These differing perspectives make the discussion of risk very challenging and also made the agreement of acceptability of risk, against predefined acceptability criteria, difficult. While current risk management standards address the processes which are required to be implemented they do not outline in detail how these processes should be implemented or discuss the potential disconnect which exists between risk management stakeholder groups when risk is discussed. 


\section{IMPROVING COMMUNICATION IN RISK MANAGEMENT}

\section{A. Domain Focused Approach to Risk Management Communication}

This paper proposes an approach that can be used to address the communication issues between different risk management stakeholders. This approach uses the Three Amigos technique which has been used in Behaviour Driven Development as a means to communicate between stakeholders and, to provide domain specific context, integrates the use of the SIMPLE tool to simplify the relevant medical terminology. The SIMPLE tool is used during the risk management process to ensure that the context in which the HITS operates, including medical device use, clinical workflows and patient safety impact, is understood by all risk management stakeholders when analysing and evaluating risk, when examining and selecting risk control measures and when ascertaining whether or not residual risk is within risk acceptability criteria. The use of the SIMPLE tool allows for seamless integration of discussion of complex medical terminology that can be understood by non-clinical risk management stakeholders in the context of the use of HITS and potential impact to patients arising from the use of HITS.

\section{B. Communication based on 'Three Amigos' and SIMPLE}

Driven by the idea of Behaviour Driven Development (BDD), we investigate and propose the "Three Amigos" approach. BDD is a software engineering methodology invented in the early to mid-2000s which uses acceptance tests to provide the starting point for the software design flow and serves as a basis for the communication between designers and stakeholders [14]. In order to facilitate communication among stakeholders, BDD uses a technique where "three amigos", consisting of various members of the project team, generally the product owner, the developer and the tester, use examples in natural language to develop acceptance tests which are then used as a basis to define requirements for the development of the final product.

Due to the demonstrated ability of the Three Amigos approach to improve communication between disciplines and its use as a risk analysis methodology for software systems [15], this paper proposes that this approach is used as a means to improve communication between various risk management stakeholders during the risk management process. This paper proposes combining the Three Amigos approach used in BDD with the use of the SIMPLE tool in order to ensure that nonclinical risk management stakeholders can gain an understanding of the impact of risk on patient safety as well as the effectiveness and security of HITS. The use of SIMPLE in this context is discussed in the following section.

\section{1) SIMPLE Description}

When dealing with health information, patients, or nonexperts in general, employ their knowledge base characterized by informal terms rather than medical jargon and, thus, they often find medical texts difficult to understand [16]-[18]. In fact, the comprehension of a medical text requires semantic and syntactic abilities that can differ from one person to another, depending on his/her literacy level. When nonexperts encounter a difficulty in understanding they usually surf the Internet for terms explanation and this can be a very time consuming and dispersive task.

SIMPLE is an online system for medical terminology simplification, that automatically finds the technical terms (words or combination of words) in an online medical document, translates them in simple or consumer terms and provides additional information in a simple language [16], [19], [20]. The system is based on an online multilingual medical vocabulary-thesaurus-dictionary that has been developed by integrating different online resources such as vocabularies, thesauri and dictionaries. In particular, a knowledge base has been created by using medical vocabularies to create a list of medical (technical) terms, consumer health vocabularies $(\mathrm{CHVs})$ for translating the technical terms into their consumer equivalents and medical consumer dictionaries for finding supplementary information on the terms. Notice that SIMPLE provides the consumer information 'aside' the original text leaving it intact.

2) Use of Three Amigos and SIMPLE for Discussion of Severity of Impact

The risk management process which is outlined in IEC 800011 is further decomposed into a 10 step approach in the technical report IEC TR 80001-2-1 [21]. The technical report advises that the specific use, needs, and concerns are needed in order to complete the risk estimation. This is referred to as "context" of use and includes information such as: acuity of patients; clinical workflow; clinical staffing and competencies; intended use/clinical or business use case; and clinical and business criticality of the systems/applications using the network. The technical report recommends having representation from multiple departments, including IT, biomedical engineering, clinical, and HDO management.

The proposed approach would use the Three Amigos approach to establish a risk management team which would consist of, at the minimum, a clinician, a BME and a member of the IT department. The team would follow the steps outlined as per IEC 80001-2-1. However, when considering the potential hazards, causes and unintended consequences and their resulting severity and probability of risk, the team would use the SIMPLE tool as a means to allow non-clinical risk management stakeholders, in this case IT, to more fully understand impact in the context of patient safety and the effectiveness and security of HITS.

To achieve this, BME would provide an explanation of the medical devices which are part of the HITS under consideration. In order to frame discussion of risk in the context of the HITS under consideration, the clinician would then provide an example or examples of the intended use of the device in the context of the clinical workflows in which the device would be used. These examples would include discussion of the acuity of patients, intended use/clinical use case and clinical criticality of the HITS. During this discussion, the SIMPLE tool can be used by clinicians to 
simplify medical terminology related to patient acuity and clinical use cases.

This will allow non-clinical risk management stakeholders to understand the potential impact from a patient perspective when a hazardous situation is experienced. This approach has the potential to aid IT in gaining an "understanding of clinical workflows". BMEs can provide further clarification around the configuration of these devices in the HITS allowing additional causes and hazardous situations to be identified. Through this discussion a more comprehensive understanding of the risk context may be established. IT having gained a more comprehensive understanding of the potential patient impact can then use their understanding to discuss the "complex networking concepts" in the established risk context in order to undertake a more accurate approach to risk analysis and evaluation.

The team should also consider the Three Amigo roles of "one to request, one suggest and one to protest" when following the 10 step risk management process. By using this approach when discussing the selection of risk control measure, this will allow the team to consider the potential and selected risk control measure to assess if the implementation will introduce additional risk or for residual risk to consider if this risk is within predetermined risk acceptability criteria. The risk associated with an individual HITS must be considered within the wider risk management context of the HDO. The following sections provide a practical example of the use of these techniques.

\section{3) SIMPLE and Risk Management - An Example}

We now present an example in order to show how SIMPLE can help during the discussion related to the risk management process. As discussed above, when considering the potential hazards, causes and unintended consequences and their resulting severity and probability of risk, SIMPLE can be used either by clinicians to explain, in simple terms, some examples of consequences to IT members to better understand the terminology used by the medical experts.

Let us assume, for example, that there is a discussion on medical devices and the associated potential risks. One of the medical devices being treated could be an infusion pump and its use for infusion therapy. The medical experts could talk about how infusion therapy works, which chronic diseases is used for and the potential risks for patients. We can then assume that there is a text that describes of how infusion therapy works. For simplicity, we take a text available on the $\mathrm{Web}^{1}$ that describes which diseases it is applied to and how it is used. Fig. 1 shows the text that is inputted to SIMPLE through a simple cut and paste action.

A first look of the text shows how it contains many medical terms that non experts will very likely not know. Fig. 2 and Fig. 3 show the same text, after being processed by SIMPLE.

\footnotetext{
1 "How it Works" section taken by https://medicorx.com/what-to-expectfrom-infusion-therapy/
}

The medical/technical terms are highlighted and an icon info button appears next to them. By clicking on the info button, the consumer term and/or explanation is shown. In particular, Fig. 2 shows the consumer information about the infusion therapy itself and Fig. 3 shows the consumer information about the Crohn's disease that is one of the chronic diseases the infusion therapy is applied to.

Insert text

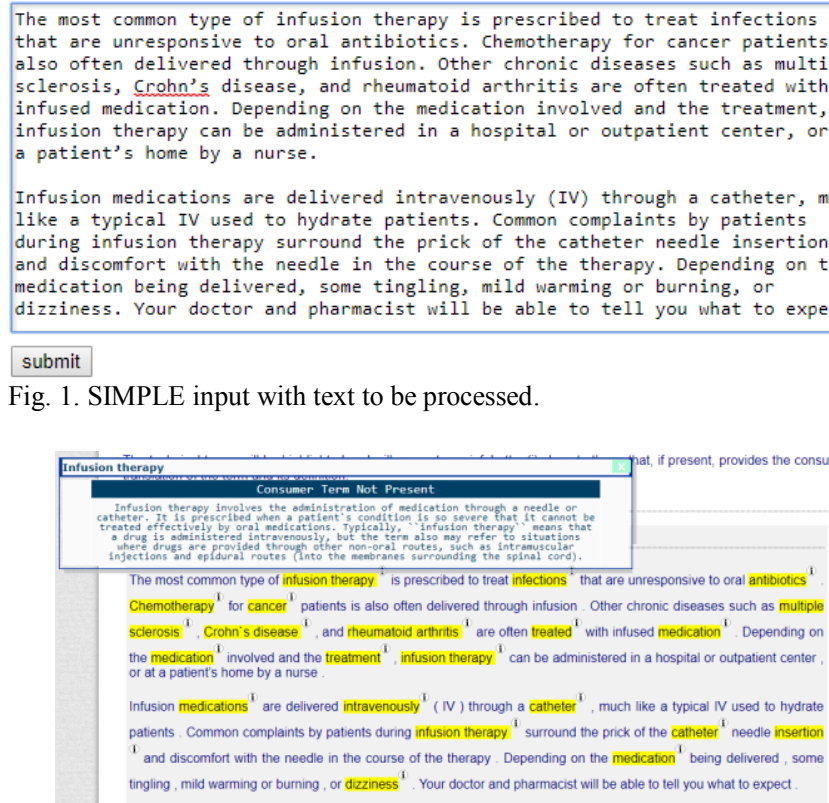

Fig. 2. SIMPLE output with info on infusion therapy.

Notice how SIMPLE can, flawlessly, be integrated into the risk management discussion providing, in real time, all the consumer information the non-experts need to follow the discussion and avoiding the burden and dispersion that a generic search engine, like Google, can provide if used to search for each term separately.

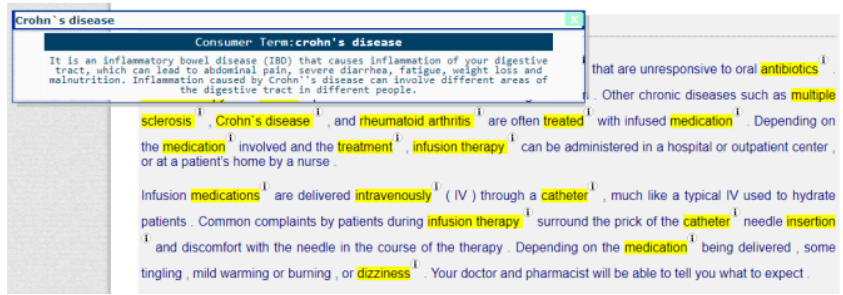

Fig. 3 SIMPLE output with info on Crohn`s disease.

4) Integrating Three Amigos and SIMPLE into the Risk Management Process

In order to fully integrate the SIMPLE tool into the risk management process, the tool is used in conjunction with the 10 step risk management process as described in IEC 80001$2-1$. The technical report provides practical example of the use of the risk management process for a small set of applicable hazardous situations and causes for each of these scenarios. This section adapts one such practical example to show how the use of the SIMPLE tool could be integrated into the 10 step risk management process, how the Three Amigos 
approach can be used to ensure representation of various risk management stakeholder groups and shows how the SIMPLE tool can be used to improve communication among them.

Context: In our example an infusion pump is being used to deliver a medication for the treatment of inflammation associated with Crohn's disease through infusion therapy. The infusion therapy is being delivered in a hospital. The acuity of patients being treated extends from less acute to complex critically ill patients.

Network Under Analysis: A 802.11 wireless area network (WLAN) covers the entire hospital and uses the $802.11 \mathrm{a} / \mathrm{b} / \mathrm{g}$ $(2.4 \& 5 \mathrm{GHz})$ band. The smart infusion pump being used for treatment of Crohn's disease is linked to the hospitals electronic health record system. The ward where the treatment is performed is located near the main kitchen, which uses high power commercial microwave ovens. The hospital also uses cordless DECT (Digital Enhanced Cordless Telecommunication) telephones in the $2,4 \mathrm{GHz}$ band.

\section{Step Risk Management Process:}

Step 1: Identify Hazards: The hazards that are identified are listed below:

HAZ01: Complete loss of connectivity.

HAZ02: Intermittent connectivity.

Step 2: Identify causes and resulting Hazardous Situations:

C01: RF interference from a microwave oven causes immediate loss of connectivity between client device and WAP (Wireless Access Point).

C02: RF interference from DECT phones causes intermittent loss of connectivity between client device and WAP.

C03: Too many client devices cause WAP overload, causing intermittent data loss.

The following Hazardous Situations are identified:

HSO1: Clinician is unaware of patient in need of treatment. Delay in treatment due to loss of data (alarms are not received by the clinician). (from Cause $\mathrm{C} 01, \mathrm{C} 02$ or $\mathrm{C} 03$ ).

Step 3: Determine unintended consequences and estimate the potential severities. The technical report provides severity scales that can be used to rank the severity of the impact on the patient and advises that severity estimation is based on knowing the acuity of the patient. The FDA has provided Infusion Pump Risk Reduction Strategies for Facility Administrators and Managers [22] and note that this includes multidisciplinary risk management stakeholders. In order for IT risk management stakeholders to be able to participate in the estimation of the severity of risk of the identified unintended consequences, they need to understand the acuity of the patients being treated and the role of the medical devices in the care processes which are used to treat these patient. As demonstrated in the previous section, by using the SIMPLE tool medical terminology surrounding the acuity of these patients and the associated care processes can be simplified leading to a greater understanding of the risk involved in the treatment of these patients and the severity of any unintended consequences associated of the identified hazardous situations.
In our example, because the acuity of the patients can vary widely and they are not under local/direct observation by a clinician, loss of real-time data for high acuity patients could lead to severe injury. The technical report notes that risk mitigations can be customized based on the acuity of the patients. In this case the severity associated with high acuity patient is considered to be catastrophic.

Step 4: Estimate the probability of the unintended consequence. In this example, we are estimating the probability that any of the causes listed above lead to the unintended consequences stated above with specified severity would be remote.

Step 5: Evaluate risk against pre-determined risk acceptability criteria. The initial risk level in this example was determined to be high based on the probability and severity determined in Steps 3 and 4.

Step 6: Identify and document proposed Risk Control measures and evaluate individual Residual Risk. In this case, Risk Control measures were identified as follows:

- $\quad$ Replace the old microwave oven effectively reducing the RF emissions because newer units are better shielded.

- Design the capacity of the network to overprovision the number of WAPs in an area such that fewer clients are serviced by a single WAP.

- To reduce the probability of severe injury, a clinician attends patients above a pre-determined acuity level effectively reducing the potential maximum severity of the injury.

- Note that no mitigation was selected specifically for Cause 2 low probability of occurrence and low practicability of mitigation (remove all DECT phones).

As the remaining steps are focused on implementing and verifying the effectiveness of risk control measures, they are not enhanced by the use of the SIMPLE tool and therefore are not discussed further in this section. Following the 10 step process, with enhanced communication through the use of the SIMPLE tool, ensures that one of the current barriers to the adoption of IEC 80001-1, that Information Technology (IT) and Biomedical Engineering (BME) departments are not aligned, is addressed.

\section{CONCLUSIONS AND FUTURE WORK}

The proposed approach has been developed to address the communication issues that are experienced by multidisciplinary risk management stakeholder in the analysis and evaluation of risk. In particular, the techniques described focus on the difficulties that are experienced by non-clinical risk management stakeholders in understanding the complex medical terminology during the discussion and estimation of the severity of risk. An Agile software development technique is combined with the use of a tool which can simplify complex medical terminology in order to provide a better means for non-clinical risk management stakeholder to participate in the estimation of the severity of risk. The proposed approach aims to provide a greater and more holistic understanding of the risk management context of HITS as defined in IEC TR 
80001-2-1 which is conducted as part of the risk management process defined in IEC 80001-1.

SIMPLE has already proven efficiency in identifying the medical terms and providing consumer translations and explanations. Nevertheless, it can be improved by expanding the medical vocabulary, thesaurus and dictionary in order to increase, respectively, the number of medical terms, consumer terms and consumer definitions also in relation to the specific context on risk management of HITS that has been treated in this paper. Moreover, considering that, as shown above, also medical experts have difficulties to understand the terms of IT experts, SIMPLE can be expanded to work as a bidirectional tool by allowing not only non-medical experts to understand medical terminology but, also, non-IT experts to understand IT technology. To this end, we plan to add vocabularies, thesauri and dictionaries of different disciplines, such as IT, so to allow text simplification and facilitate understanding by non-experts in those disciplines. We also plan to facilitate the search of specific medical information on the Internet should this be needed [23].

Future work in this area will focus a pilot implementation of the proposed approach within a HDO. The pilot implementation will be performed be framing a discussion of risk for a planned HITS implementation. The research team performing this research has previously performed pilot implementations of risk management frameworks within HDOs as part of an Action Design Research Approach to the development of ISO TR 80001-2-7. While the research is at an early stage the approach is being proposed in response to an issue which has been identified by a survey conducted by members of the international standard development community. While work on the revision of the IEC 80001-1 seeks to address some of the barriers to the adoption of the standard, it does not directly address the communication issues between risk management stakeholders. This research will use existing research collaborations with HDOs and members of the international standard community to establish the efficacy of the proposed approach.

\section{ACKNOWLEDGMENT}

This work was partially supported with the financial support of the Science Foundation Ireland grant 13/RC/2094 and co-funded under the European Regional Development Fund through the Southern \& Eastern Regional Operational Programme to Lero - the Irish Software Research Centre (www.lero.ie). Moreover, it was partially supported by the European Union's Horizon 2020 research and innovation programme under the Marie Skłodowska-Curie grant agreement No 754489.

\section{REFERENCES}

[1] West Health Institute, "The Value of Medical Device Interoperability - Improving patient care with more than $\$ 30$ billion in annual health care savings," 2013.

[2] S. F. Whitehead and J. M. Goldman, "Getting Connected for Patient Safety How Medical Device 'Plug-and-Play' Interoperability Can Make a Difference," Patient Saf. Qual. Healthc., no. January/February 2008, 2008.
[3] K. K. Venkatasubramanian, S. K. S. Gupta, R. P. Jetley, and P. L. Jones, "Interoperable Medical Devices - Communication Security Issues," IEEE Pulse, vol. Sept/Oct 2, 2010.

[4] E. H. Wagner, B. T. Austin, C. Davis, M. Hindmarsh, J. Schaefer, and A. Bonomi, "Improving chronic illness care: translating evidence into action," Health Aff., vol. 20, no. 6, pp. 64-78, 2001.

[5] C. Hoffman and D. Rice, "Chronic care in America: A 21st century challenge," Princeton, NJ Robert Wood Johnson Found., 1996.

[6] J. Comstock, "14M networked medical devices to ship by 2018," mobi health news, 2013. [Online]. Available: http://mobihealthnews.com/28295/14m-networked-medicaldevices-to-ship-by-2018/.

[7] T. Gee, "Medical Device Networks Trouble Industry," 2008. [Online]. Available: http://medicalconnectivity.com/2008/12/18/medical-devicenetworks-trouble-industry/.

[8] S. Eagles, "An Introduction to IEC 80001: Aiming for Patient Safety in the Networked Healthcare Environment," IT Horizons, vol. $2008,2008$.

[9] S. R. Rakitin, "Networked Medical Devices: Essential Collaboration for Improved Safety," AAMI.org, 2009.

[10] IEC, "IEC 80001-1 - Application of Risk Management for ITNetworks incorporating Medical Devices - Part 1: Roles, responsibilities and activities." International Electrotechnical Commission, Geneva, Switzerland, 2010.

[11] ISO, "ISO 31000:2009 Risk management -- Principles and guidelines." Geneva, Switzerland, 2009.

[12] S. T. MacMahon, T. Cooper, and F. McCaffery, "Revising IEC 80001-1: Risk management of health information technology systems," Comput. Stand. Interfaces, 2018.

[13] F. J. Hegarty, S. T. MacMahon, P. Byrne, and F. McCaffery, "Assessing a hospital's medical IT network risk management practice with 80001-1," Biomed. Instrum. Technol., vol. 48, no. 1, 2014.

[14] M. Soeken, R. Wille, and R. Drechsler, "Assisted behavior driven development using natural language processing," Lect. Notes Comput. Sci. (including Subser. Lect. Notes Artif. Intell. Lect. Notes Bioinformatics), vol. 7304 LNCS, pp. 269-287, 2012.

[15] J. Wang, "Modeling Techniques for a Risk Analysis Methodology for Software Systems," no. June, pp. 1-42, 2011.

[16] M. Alfano, B. Lenzitti, G. Lo Bosco, and D. Taibi, "Development and Practical Use of a Medical Vocabulary-Thesaurus-Dictionary for Patient Empowerment," in Proceedings of the 19th International Conference on Computer Systems and Technologies, 2018, pp. 8893.

[17] G. Leroy, J. E. Endicott, O. Mouradi, D. Kauchak, and M. L. Just, "Improving perceived and actual text difficulty for health information consumers using semi-automated methods," in AMIA Annual Symposium Proceedings, 2012, vol. 2012, p. 522.

[18] M. Kvist and S. Velupillai, "Professional language in swedish radiology reports-characterization for patient-adapted text simplification," in Scandinavian Conference on Health Informatics 2013, Copenhagen, Denmark, August 20, 2013, 2013, pp. 55-59.

[19] M. Alfano, B. Lenzitti, G. Lo Bosco, and V. Perticone, "An Automatic System for Helping Health Consumers to Understand Medical Texts.," in HEALTHINF, 2015, pp. 622-627.

[20] M. Alfano, B. Lenzitti, G. Lo Bosco, and V. Perticone, "Facilitating text understanding for e-learning users," in International COnference on E-Learning e-Learning'14, 2014.

[21] IEC, "PD IEC/TR 80001-2-1:2012 - Application of risk management for IT-networks incorporating medical devices - Part 2-1: Step by step risk management of medical IT-networks; Practical applications and examples." International Electrotechnical Commission, Geneva, Switzerland, 2012.[22

US Food and Drug Administration, "Infusion Pump Risk Reduction Strategies for Facility Administrators and Managers," 2018.

M. Alfano, B. Lenzitti, D. Taibi, M. Helfert. "Facilitating access to health web pages with different language complexity levels". In Proc. of the 5thInternational Conference on Information and Communication Technologies for Ageing Well and e-Health (ICT4AWE 2019). 2019. 